\title{
Physician variation in opioid prescribing: the importance of sex and gender
}

\author{
Paula Rochon, ${ }^{1,2,3}$ Parya Borhani, ${ }^{1,4}$ Jennifer Akerman, ${ }^{1,5}$ \\ Anamika Mishra ${ }^{6}$
}

${ }^{1}$ Women's Age Lab and Women's College Research Institute, Women's College Hospital, Toronto, Ontario, Canada

${ }^{2}$ Department of Medicine and Institute of Health Policy, Management and Evaluation, University of Toronto, Toronto, Ontario, Canada

${ }^{3}$ RTOERO Chair, Geriatric Medicine, University of Toronto, Toronto, Ontario, Canada ${ }^{4}$ Dalla Lana School of Public Health, University of Toronto, Toronto, Ontario, Canada ${ }^{5}$ Dalhousie University, Halifax, Nova Scotia, Canada

${ }^{6}$ Western University, London, Ontario, Canada

\section{Correspondence to} Dr Paula Rochon, Women's College Hospital, Toronto, Ontario M5S 1B2, Canada; paula.rochon@wchospital.ca

Accepted 19 August 2021

\section{SLinked}

- http://dx.doi.org/10.1136/ bmjqs-2021-013503

Check for updates

(c) Author(s) (or their employer(s)) 2021. No commercial re-use. See rights and permissions. Published by BMJ.

To cite: Rochon $\mathrm{P}$, Borhani $\mathrm{P}$, Akerman J, et al. BMJ Qual Saf Epub ahead of print: [please include Day Month Year]. doi:10.1136/

bmjqs-2021-013697
Concern about the link between opioid prescribing and preventable adverse drug events has led to a series of initiatives to reduce opioid use, with opioids identified as one of three high-priority drug classes targeted to reduce patient harms in the United States (US)'s National Action Plan for Adverse Drug Event Prevention. ${ }^{1}$ Variation in opioid prescribing practices by physicians has been observed, yet the reasons why these differences exist remain largely unknown. A better understanding of these differences may help to improve prescribing practice for opioids. Sex and gender considerations in opioid prescribing practices have not been well studied and may help address this important knowledge gap.

There is some evidence to suggest that sex and gender of physicians can affect prescribing practices for older adults. ${ }^{2}$ Patient gender has also been related to the experience of health conditions, healthseeking behaviours and medication use. $^{3-5}$ Sex (biologic), a term describing the physical characteristics and biological attributes of males, females and intersex individuals, influences biological manifestations of medical conditions and responses to drug therapy. ${ }^{5}$ In contrast, gender (sociocultural) refers to the socially constructed norms, behaviours and roles associated with being a man, woman or gender diverse person. ${ }^{6}$ While these concepts are related, they are often incorrectly interchanged. Considering sex and gender, and how they intersect with key identity factors such as age, culture, race and ethnicity, is an analytical approach ${ }^{7}$ that can be applied to systematically explore the influence of sex and gender on prescribing practices, revealing potentially important differences or trends that would otherwise remain obscured. Such an approach provides the opportunity to inform prescribing practices in general, and pain management strategies in particular. This will allow healthcare provision to be tailored to the unique needs of women, men and gender diverse people, including those in different age groups, acknowledging the evolution of health and medication needs across the life span.

The study by Tamblyn and colleagues, ${ }^{8}$ published in this edition of the journal, underscores the utility and value of considering patient and provider sex along with other key identity factors such as age, race and culture in all clinical research. This study also presents the opportunity to consider the wider role of patient and physician gender on prescribing, and why the inclusion of both sex and gender may be essential to uncovering important variations in clinical practice, and to capturing the diversity of health needs and experiences in patient populations.

In their study, Tamblyn ${ }^{8}$ set out to explore the impact of physician characteristics, including clinical competence, specialty and country of origin, on opioid prescribing for chronic noncancer pain. The authors examined the opioid prescribing patterns of a cohort of international medical graduates in the US who completed their Clinical Skills Assessment requirement for the Educational Commission for Foreign Medical Graduates between 1998 and 2004. They were managing older patients covered by the Medicare part D drug insurance programme who were receiving care in an ambulatory setting for chronic non-cancer pain in 2014 and 2015. Logistic and linear regression were used to explore the association of the prescribing physician's characteristics with opioid prescribing and the doses prescribed. 
Outcomes measured in the study included opioid prescribing within 90 days of the clinical evaluation, whether the patient received a non-opioid intervention (eg, physiotherapy treatment or a non-steroidal anti-inflammatory drug) prior to the opioid prescription, and the opioid dose that was prescribed. ${ }^{8}$ The former outcome is particularly important, as opioids are not first-line therapy for chronic pain. ${ }^{9}$

Notably, even though it was not identified as one of the primary variables of interest, the Tamblyn study ${ }^{8}$ revealed the importance of routinely stratifying data by sex. Further, the study underlines the importance of the collection and use of sex and age disaggregated data to better understand health status. ${ }^{10}$ More specifically, this study illustrates why it is important to consider provider sex in opioid prescribing, as well as genderrelated sociocultural factors. First, there are important physician and patient factors that relate to their sex and others that are gender related. Most (61\%) of the prescribing physicians in this study were men. This is in part because medicine itself is gendered. ${ }^{11}$ While the proportion of female physicians has grown substantially over the past few decades, they remain underrepresented in most specialties, especially those that are higher paying, including some of those explored within the present study. ${ }^{11} 12$

Women are more likely than men to experience the chronic conditions that cause pain. ${ }^{13}$ The most common chronic non-cancer conditions being managed in this study were back and neck pain, migraine/headaches, rheumatoid or osteoarthritis, and neuropathic pain. Each of these conditions occur more commonly in older women than in older men. For example, compared with men of the same age, women aged 65 years and older in Canada are 1.9 times more likely to have been diagnosed with rheumatoid arthritis and are 1.4 times more likely to have been diagnosed with osteoarthritis. ${ }^{14}$ Migraine headaches are experienced by almost twice as many women as men $(7.4 \%$ of women compared with $3.4 \%$ of men $)^{10}$ and neuropathic pain is similarly more prevalent among women. ${ }^{13}$ The predominance of older women experiencing these chronic non-cancer pain conditions may be one reason why more than $66 \%$ of the older patients being managed for chronic pain in the Tamblyn study were women. Unless these differences are examined using sex and age disaggregated data, important patterns in the characteristics of the prescribers and the differences in pain experienced by older women and men will remain hidden in the data.

A second salient finding of this study was that the odds of prescribing an opioid for non-cancer chronic conditions was $11 \%$ higher for male physicians (OR $1.11,95 \%$ CI 1.03 to 1.19$).{ }^{8}$ Further, for every $10 \%$ increase in the clinical encounter score (used to measure clinical competence), the odds of prescribing an opioid decreased by $16 \%$ for female physicians (OR $0.84,95 \%$ CI 0.75 to 0.94 ), but not for male physicians (OR 0.99 , 95\% CI 0.92 to 1.07 ). ${ }^{8}$ These findings align with the existing literature that reports on correlations between physician gender-related sociocultural factors and prescribing behaviour, patient care and clinical outcomes. Female physicians have been shown to prescribe medications at lower doses than male physicians. ${ }^{15}$ The initiation of medications at low doses, using the 'start low, go slow approach', is a practice from geriatric medicine that minimises the risk of harm in older adults, as adverse events are often dose related. ${ }^{16}$ This was illustrated in a study of the initiation of drug therapy for the management of dementia. Female prescribers were more likely than their male counterparts to initiate cholinesterase inhibitor therapy at a lower-than-recommended dose and for a shorter duration. ${ }^{15}$ Previous literature on the prescribing of opioids similarly finds that female physicians prescribe opioids more sparingly than their male counterparts. ${ }^{17}$ When it comes to patient-clinician interactions, female physicians have been characterised as providing more patient-centred and empathetic care. ${ }^{18}{ }^{19}$ Compared with their male counterparts, they have been shown to spend more time with patients, engage in more communicative and active partnerships, and provide more psychosocial support and counselling. ${ }^{19}$ Female physicians have also been shown to adhere more closely to clinical guidelines ${ }^{20}$ and practise more evidence-based medicine. ${ }^{21}$ These differences in care delivery and treatment provision may be linked to the more conservative prescribing practices of female physicians demonstrated in the present study and elsewhere. ${ }^{15} 17$

The differences in opioid prescribing practices between male and female physicians observed in the Tamblyn study are not isolated to pain management or opioid prescribing. Rather, this study contributes to recent evidence from different clinical settings and specialties, suggesting that patients cared for by female physicians may have better clinical outcomes compared with their male colleagues. For instance, when matched for patient, surgeon and hospital characteristics in a large population-based cohort study, patients of female surgeons identified as having 1 of 25 index procedures were found to experience lower risk of short-term postoperative death than those cared for by male surgeons. ${ }^{22}$ Similarly, female internists treating older adults hospitalised with a medical condition were found to provide significantly better outcomes than their male colleagues in terms of 30-day mortality and readmission rates. ${ }^{23}$

Data on physician and patient sex or genderrelated sociocultural factors are often not reported on or described in research studies, making further synthesis of findings through meta-analysis difficult. ${ }^{24}$ Consistent reporting of this information can allow for aggregation of data and establishment of stronger correlations between prescriber sex and gender, and clinical outcomes. 
Finally, considering a sex-based and gender-based analytical approach that includes an intersection with cultural factors for both patients and prescribers may be key to a better understanding of opioid prescribing and pain management. Gender-based psychosocial patient factors have been related to behavioural responses and expressions of perceived pain, which often reflect societal norms. ${ }^{25}$ As a social construct, gender is understood to be context specific and thus varying cultural expectations for pain management, potentially linked to country of origin, may influence how a patient experiences pain and a physician's likelihood of opioid prescribing for common pain problems. In the present study, Tamblyn found that while the prescriber's country of origin did not influence the odds of opioid prescribing, US and Canadian physicians, both men and women, prescribed opioids at higher doses. ${ }^{8}$ Therefore, the potential influence of cultural norms and gender in relation to clinical treatment and diagnosis of pain may also reveal disproportionate cross-national impacts that would otherwise remain hidden.

This study has highlighted the importance of considering patient and prescriber sex, gender and other key identity factors including age and culture, in all research studies in order to better inform clinical care. Given the risks associated with potentially inappropriate opioid use in older adults, it is worth exploring further how the more cautious practices of female physicians could offer a learning opportunity to optimise health outcomes for all.

Funding The authors have not declared a specific grant for this research from any funding agency in the public, commercial or not-for-profit sectors.

Competing interests None declared.

Patient consent for publication Not required.

Provenance and peer review Commissioned; internally peer reviewed.

\section{REFERENCES}

1 U.S. Department of Health and Human Services, Office of Disease Prevention and Health Promotion. National action plan for adverse drug event prevention. Washington, DC, 2014. https://health.gov/sites/default/files/2019-09/ADE-Action-Plan508c.pdf

2 Mishra A, Read SH, Rochon PA. Influence of physician sex and gender on prescribing practices among older adults. J Am Geriatr Soc 2020;68:2764-7.

3 Morgan R, George A, Ssali S, et al. How to do (or not to do)... gender analysis in health systems research. Health Policy Plan 2016;31:1069-78.

4 Vlassoff C, Garcia Moreno C. Placing gender at the centre of health programming: challenges and limitations. Soc Sci Med 2002;54:1713-23.

5 Regitz-Zagrosek V. Sex and gender differences in pharmacology. Berlin, Heidelberg: Springer, 2012.

6 World Health Organization. Gender and health. Available: https://www.who.int/health-topics/gender\#tab=tab_1 [Accessed 17 Jun 2021].
7 Canadian centre on substance use and addiction. sex- and gender-based Analysis +. Available: https://www.ccsa.ca/sexand-gender-based-analysis [Accessed 17 Jun 2021].

8 Tamblyn. Tracking number: bmjqs-2021-013503

9 U.S. Department of Health and Human Services. Centers for disease control and prevention. guideline for prescribing opioids for chronic pain. Available: https://www.cdc.gov/ drugoverdose/pdf/prescribing/Guidelines_Factsheet-a.pdf [Accessed 21 Jun 2021].

10 UN decade of healthy ageing. 2021-2030. Available: https:// www.who.int/initiatives/decade-of-healthy-ageing [Accessed 25 Jun 2021].

11 Cohen M, Kiran T. Closing the gender pay gap in Canadian medicine. CMAJ 2020;192:E1011-7.

12 Association of American Medical Colleges. Active physicians by sex and specialty, 2019. Available: https://www.aamc.org/ data-reports/workforce/interactive-data/active-physicians-sexand-specialty-2019 [Accessed 17 Aug 2021].

13 Fillingim RB, King CD, Ribeiro-Dasilva MC, et al. Sex, gender, and pain: a review of recent clinical and experimental findings. J Pain 2009;10:447-85.

14 The public health agency of Canada. aging and chronic diseases: a profile of Canadian seniors. Available: https:// www.canada.ca/en/public-health/services/publications/diseasesconditions/aging-chronic-diseases-profile-canadian-seniorsexecutive-summary.html [Accessed 17 Jun 2021].

15 Rochon PA, Gruneir A, Bell CM, et al. Comparison of prescribing practices for older adults treated by female versus male physicians: a retrospective cohort study. PLoS One 2018;13:e0205524.

16 Halter JB, Ouslander JG, Studenski S. Hazzard's Geriatric Medicine and Gerontology. 7th ed. New York: McGraw Hill Education, 2017.

17 Harrison CM, Charles J, Henderson J, et al. Opioid prescribing in Australian general practice. Med J Aust 2012;196:380-1.

18 Howick J, Steinkopf L, Ulyte A, et al. How empathic is your healthcare practitioner? A systematic review and meta-analysis of patient surveys. BMC Med Educ 2017;17:136.

19 Roter DL, Hall JA. Physician gender and patient-centered communication: a critical review of empirical research. Annu Rev Public Health 2004;25:497-519.

20 Baumhäkel M, Müller U, Böhm M. Influence of gender of physicians and patients on guideline-recommended treatment of chronic heart failure in a cross-sectional study. Eur J Heart Fail 2009;11:299-303.

21 Berthold HK, Gouni-Berthold I, Bestehorn KP, et al. Physician gender is associated with the quality of type 2 diabetes care. $J$ Intern Med 2008;264:340-50.

22 Wallis CJ, Ravi B, Coburn N, et al. Comparison of postoperative outcomes among patients treated by male and female surgeons: a population based matched cohort study. BMJ 2017;359:j4366.

23 Tsugawa Y, Jena AB, Figueroa JF, et al. Comparison of hospital mortality and readmission rates for Medicare patients treated by male vs female physicians. JAMA Intern Med 2017;177:206-13.

24 Etherington N, Deng M, Boet S, et al. Impact of physician's sex/gender on processes of care, and clinical outcomes in cardiac operative care: a systematic review. BMJ Open 2020;10:e037139.

25 Templeton KJ. Sex and gender issues in pain management. $J$ Bone Joint Surg Am 2020;102 Suppl 1:32-5. 